\title{
Lack of Haptic Feedback Is Replaced by More Developed Visual Sense during Robotic Myomectomy
}

\author{
Hye-Sung Moon, Eunhye Cho, Hae Kyung Yoo
}

Department of Obstetrics and Gynecology, Gynecologic Oncology Branch \& Robot Surgery Center, Ewha Womans University College of Medicine, Seoul, Korea

In the reproductive age, many women have several uterine myomas and present with abnormal uterine bleeding, dysmenorrhea, and occasionally infertility. There are three surgical approaches to perform myomectomy, including robotic-assisted, laparoscopic, and abdominal myomectomy. Compared to laparoscopic procedures, robotic myomectomy allows free approach of myoma bases using fine instruments and endoscopes. Fine uterine wall sutures can be performed layer-by-layer with robots. However, robotic surgery is difficult to perform because there is no sense of touch during the operation. We report two clinical myomectomy cases with replaced lack of haptic feedback during robot surgery. The patients received robotic myomectomy with/without right ovarian cystectomy and adhesiolysis. Sixty-five leiomyomas were removed in case 1. Forty-six leiomyomas were removed in case 2. Lack of haptic feedback is replaced by more developed visual sense during robot myomectomy of multiple tiny intramural myomas, and robotic surgery can be performed much more effectively even in complicated cases. (Ewha Med J 2019;42(2):20-23)
Received September 27, 2018

Revised January 14, 2019

Accepted March 21, 2019

\section{Corresponding author}

Hye-Sung Moon

Department of Obstetrics and Gynecology,

Ewha Women Cancer Center, Ewha

Womans University College of Medicine,

25 Magokdong-ro 2-gil, Gangseo-gu, Seoul 07804, Korea

Tel: 82-2-6986-1678, Fax: 82-2-2647-9860

E-mail: mhsmhs@ewha.ac.kr

\section{Key Words}

Uterine myomectomy; Myoma; Robotic surgical procedures

\section{Introduction}

In the reproductive age group, many women have several uterine myomas that present with abnormal uterine bleeding, low abdominal discomfort, and occasionally infertility [1]. Clinically, asymptomatic uterine myomas are diagnosed in $25 \%$ to $40 \%$ of all women and the incidence of myomas increases with age [1]. Although uterine myomas tend to have no positive influence on fertility, myomectomy is considered to maintain fertility. There are three types of surgical approach to perform myomectomy, including robotic-assisted, laparoscopic, and abdominal myomectomy. These surgical approaches have different advantages and disadvantages regarding patient recovery and fertility [2].

In performing myomectomy, uterine scar repair is the most important factor affecting fertility outcomes. With open myomectomy procedures, most myomas can be removed by palpating uterine walls. However, patients suffer more from postoperative wound pain and have a large scar.

To avoid postoperative pain, patients often desire minimally invasive surgeries even with multiple large myomas. Other minimally invasive surgical techniques include laparoscopic or robotassisted laparoscopic myomectomy [2]. However, in laparoscopic myomectomy, it is difficult to approach the deep base origins of large myomas. It is also difficult to perform layer-by-layer sutures of the uterine wall and remove tiny and deep seated myoma nodules. Compared to laparoscopic procedures, robotic myomectomy uses fine wristed instruments with more magnification to allow approach of various myoma bases, performance

This is an Open Access article distributed under the terms of the Creative Commons Attribution Non-Commercial License (http://creativecommons.org/licenses/by-nc/4.0) which permits unrestricted non-commercial use, distribution, and reproduction in any medium, provided the original work is properly cited. 
of fine sutures of the uterine wall, and decrease the incidence of uterine rupture in pregnancy.

We have several selection criteria for robot myomectomy. One of the selection criteria was the desire of patients to receive robot surgery. And candidates for robotic myomectomy are the patients who have deeply seated myomas that are located at more than half of the myometrium and have multiple myomas including small myomas spread in the whole uterus. Robot myomectomy was also offered to patients who want to preserve fertility.

However, surgeons may experience difficulty performing robotic surgery because of the lack of touch sense. With enhanced visual sense during the operation, lack of haptic feedback can be replaced during robotic surgery. Visual sense can be developed with more experience in robotic surgery and performance of more complicated robotic surgery.

According to previous studies, the learning curve to stabilize operative times for the various surgical procedures in women requiring benign gynecolologic interventions is 50 cases [3]. There are other findings that 50 cases are required to develop a surgeon's abilities to find myomas in a visual sense and discover deeply buried myomas. The learning curve could be reached rapidly with high-volume surgical centers. It also depends on the surgeon's surgical skill [4].

Herein, we report two clinical cases of robotic myomectomy in cases of multiple tiny myomas to show that lack of haptic feedback in robotic surgery can be replaced by the visual sense.

\section{Case}

\section{Case 1}

A 30-year-old woman (body mass index $20.48 \mathrm{~kg} / \mathrm{m}^{2}$, gravida 0 , para 0 ) had menorrhagia and back pain for 1 to 2 years and had underwent myomectomy 5 years before. Pelvic ultrasound and MRI revealed a $12.8 \times 9.7-\mathrm{cm}$ uterus with several intramural myomas having a maximum diameter of $8.4 \mathrm{~cm}$, almost 30 tiny myoma nodules less than $1 \mathrm{~cm}$, and an adnexal mass (Fig. 1A, B). The patient received robotic multi-site myomectomy with right ovarian cystectomy and adhesiolysis.

The uterine serosa wall was cut over uterine myoma nodules that were removed using a bipolar forceps and monopolar hook. After excising myomas, the muscle walls were repeatedly knocked. Bouncing of the instrument tips indicated that there were myoma nodules buried deep inside the muscle. All tiny myoma nodules buried inside the muscle wall were removed (Fig. 1C, D). The uterine wall was sutured in layer-by-layer
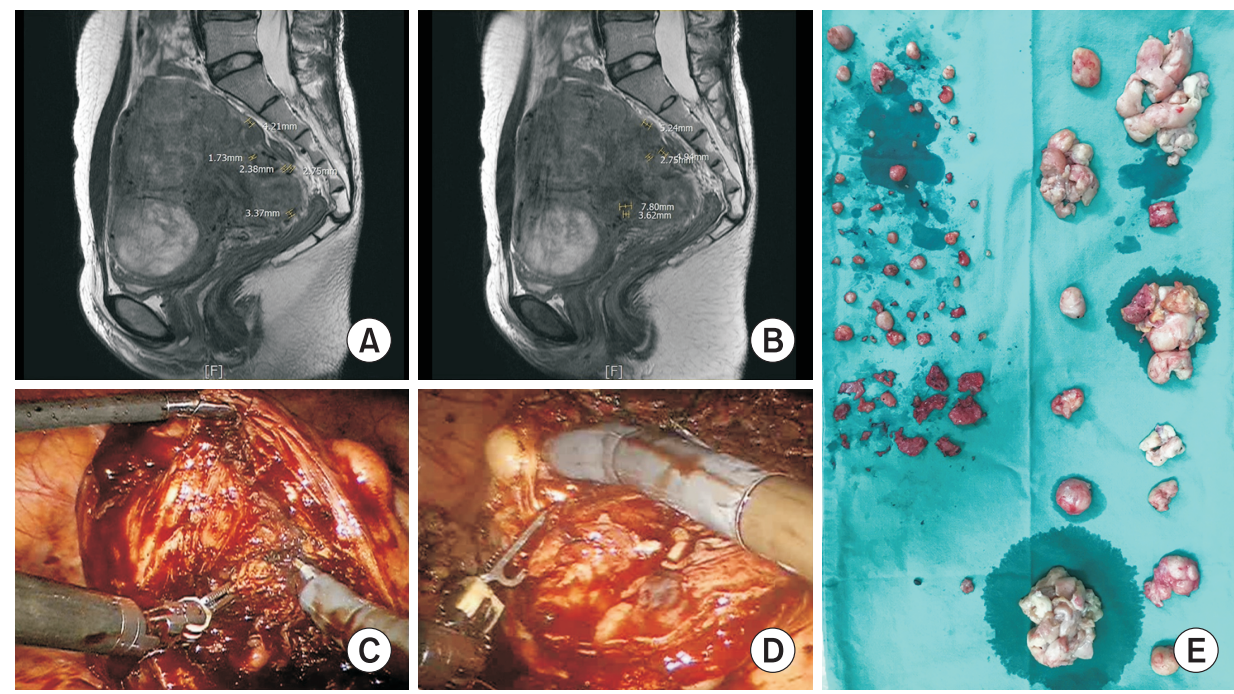

Fig. 1. Radiologic imaging and surgical findings of case 1. (A) Sagittal view of pelvis MRI finding in case 1 with measured tiny intramural myoma nodules. (B) Other sagittal view of pelvis MRI finding in case 1 with measured tiny intramural myoma nodules. (C) Deep seated tiny myoma nodules shown during robotic myomectomy. The robotic bipolar forceps tip is shorter than $1.5 \mathrm{~cm}$ in length and longer than the longest diameter of tiny myoma nodules. (D) Deep seated tiny myoma nodules were shown during robotic myomectomy. The longest diameter of myoma nodule is similar to the 1.2-cm length of robotic metzenbaum scissor's tip. (E) All myoma specimens in case 1 removed with various sizes. 
fashion with four layers using a wristed needle driver.

The excised myomas were pulled down in an extra-large endobag assisted by the bipolar forceps and wristed needle driver bilaterally. To extract myomas, the bag was opened through a wound retractor in the umbilicus, and myomas were cut and removed in pieces. The ports were reinserted with three straight and reusable trocars to clean the blood and examine the pelvic cavity. The operation time was 336 minutes and the estimated blood loss was about $900 \mathrm{~mL}$. The patient stayed in the hospital for 6 days and there was no complication except postoperative fever with a maximum of $37.8^{\circ} \mathrm{C}$ at 3 days after surgery.

There were 65 leiomyomas removed in total, and the largest one was $7 \times 6 \mathrm{~cm}$. There were 45 myomas less than $1 \mathrm{~cm}$ (Fig. 1E). The right adnexal mass was $8 \times 6 \mathrm{~cm}$, had regular margins, and was filled with transparent cystic fluid. Pathologic type was cellular leiomyoma with focal atypia with mitotic count less than $1 / 10 \mathrm{HPF}$.

\section{Case 2}

A 30-year-old woman with body mass index of $18.51 \mathrm{~kg} / \mathrm{m}^{2}$, gravida 0 , para 0 presented with history of dysmenorrhea and prolonged menstruation. Pelvic ultrasound and MRI revealed a $7.90 \times 5.44-\mathrm{cm}$ uterus with multiple myomas and 28 tiny myoma nodules less than $1 \mathrm{~cm}$ (Fig. 2A, B).

The patient received robotic multi-site myomectomy. A bi-
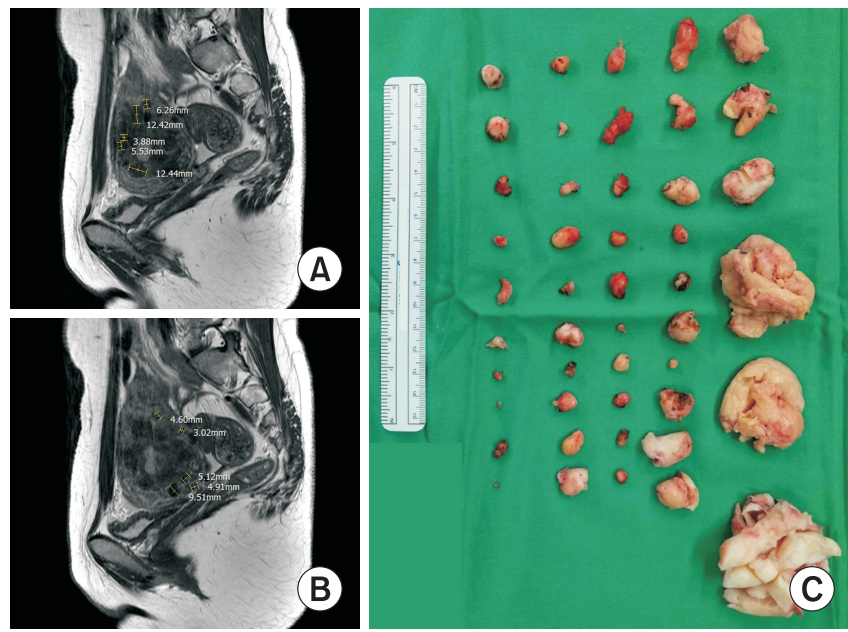

Fig. 2. Radiologic imaging and surgical findings of case 2. (A) Sagittal pelvis MRI finding of case 2 with measured tiny myoma nodules in anterior wall of uterus. (B) Other sagittal view of pelvis MRI finding in case 2 with tiny intramural myoma nodules in posterior wall of uterus. (C) All removed myoma specimens in case 2. polar forceps and monopolar hook were passed over the uterine myoma to excise. Buried myoma nodules were identified below the uterine serosa by picking up the muscle walls with bipolar forceps. All tiny myoma nodules buried inside the muscle wall were removed. The uterine wall was sutured in layer-by-layer fashion in four layers using a wristed needle driver.

The excised myoma were extracted by same manner with case 1. The operation time was 400 minutes, and the estimated blood loss was $400 \mathrm{~mL}$. She had a fever of $37.7^{\circ} \mathrm{C}$ at 2 days after surgery.

There were 46 leiomyomas removed in total, and the largest was $4 \times 3 \mathrm{~cm}$. There were 38 myomas less than $1 \mathrm{~cm}$ (Fig. 2C). Pathologic type was focal hypercellular leiomyoma with no atypia and mitotic count less than 1/10 HPF.

After surgery, the patient was followed up for two years. She was administered leuprolide acetate six times at intervals of about one month. Four months later, recurrence of fibroids was identified and there were two 1-cm-sized myomas on the uterus. Sixteen months after surgery, there were multiple myomas ranging from 1 to $3 \mathrm{~cm}$.

Both cases were included by approval of the institutional review board of Ewha Womans University College of Medicine (No. 2016-12-001-002). Written informed consents were obtained.

\section{Discussion}

In this study, robotic-assisted laparoscopic myomectomy was performed to remove 46 and 65 myomas in two patients. There were 38 and 45 tiny myoma nodules less than $1 \mathrm{~cm}$ in maximum diameter in each case. Pelvis MRI showed some myomas less than $1 \mathrm{~cm}$ without a fine border.

Recently, minimally invasive surgery techniques are mostly used in gynecologic fields. It is difficult to perform laparoscopic or robotic myomectomy to remove multiple tiny nodules seated deeply under larger myomas because there is a lack of the touch sense, especially with robotic surgery. In this study, we overcame the lack of haptic feedback during robotic surgery with more developed visual sense. Tactile images are replaced by the visual sense during the operation.

With high resolution tactile image during robotic surgery that are superior to the sense of touch, there is potential to remove tiny deep seated myoma nodules from the myometrium. $\mathrm{Re}^{-}$ 
peated knocking of uterine muscle walls and bouncing of instrument tips indicated buried myoma nodules deep inside. Surgeons could also identify buried myoma nodules below the uterine serosa by picking up the muscle walls with bipolar forceps. To develop robotic surgery skills, the visual sense is more important. Therefore, high-resolution tactile imaging that is superior to the sense of touch has potential for future biomedical applications such as robotic surgery [5].

In these two cases, the pathologic findings were cellular leiomyoma with 1 mitosis/10 HPF [6]. Among the numerous histological subtypes of leiomyoma, cellular fibroids are the most common (5\%). Histopathologic examination revealed cell abundance with a small component of connective elements, little or no atypia, absence of coagulated necrosis, and mitotic count of $\langle 5 / 10 \mathrm{HPF}$ (microscope field of view at $\times 40$ magnification). There is no symptom that specifically associated with cellular leiomyoma, and management of cellular leiomyoma does not differ from that of ordinary leiomyoma. In contrast to malignant disease, cellular leiomyoma has a favorable long-term prognosis [7].

Discussions remain regarding whether removing all myoma nodules less than $1 \mathrm{~cm}$ is required. The recurrence rate after myomectomy is associated with the number of myomas removed during surgery. Previous studies have reported that a larger number of enucleated myomas is associated with a higher recurrence rate. Thus, in these patients, larger number and size of myomas are thought to be related to higher recurrence rates [8].

The number of fibroids removed during myomectomy significantly affects fertility. Women with $>6$ fibroids removed were less likely to become pregnant and less likely to have a baby to full term compared to women with $\leq 6$ fibroids removed [1]. However, the current cases were cellular leiomyomas, which often recur. Therefore, we tried to remove all myoma nodules to prevent recurrence. A recent case series of robotic myomectomy for women with deep intramural myomas showed a pregnancy rate of $68 \%$ after surgery, although many of these pregnancies were accomplished by assisted reproductive technologies [9]. Further study is needed to investigate whether complete removal of cellular leiomyoma nodules by myomectomy positively affects reoccurrence of pregnancy.

There is no significant difference in long-term bleeding or fertility outcomes in robotic-assisted, laparoscopic, or abdominal myomectomy [2]. A previous study revealed that robotic surgery improved fibroid enucleation and layered closure of uterine incisions compared to laparoscopy; it also provided an unsurpassed level of surgeon's comfort [10]. Robotic myomectomy provides the opportunity to finely repair uterine walls layer-by-layer without injuring the endometrium when removing multiple myomas.

In conclusion, lack of haptic feedback is replaced by a more developed visual sense during robotic myomectomy of multiple tiny intramural myomas, and robotic surgery can be performed much more effectively even in complicated cases.

\section{References}

1. Shue S, Radeva M, Falcone T. Comparison of long-term fertility outcomes after myomectomy: relationship with number of myomas removed. J Minim Invasive Gynecol 2018;25:1002-1008.

2. Flyckt R, Soto E, Nutter B, Falcone T. Comparison of long-term fertility and bleeding outcomes after robotic-assisted, laparoscopic, and abdominal myomectomy. Obstet Gynecol Int 2016;2016:2789201.

3. Lenihan JP Jr, Kovanda C, Seshadri-Kreaden U. What is the learning curve for robotic assisted gynecologic surgery? J Minim Invasive Gynecol 2008;15:589-594.

4. Gargiulo AR, Srouji SS, Missmer SA, Correia KF, Vellinga TT, Einarsson JI. Robot-assisted laparoscopic myomectomy compared with standard laparoscopic myomectomy. Obstet Gynecol 2012;120:284-291.

5. Wijesinghe P, Sampson DD, Kennedy BF. Computational optical palpation: a finite-element approach to micro-scale tactile imaging using a compliant sensor. J R Soc Interface 2017;14:20160878.

6. Sikora-Szczesniak DL. Prevalence of cellular leiomyoma and partially cellular leiomyoma in postoperative samples: analysis of 384 cases. Ginekol Pol 2016;87:609-616.

7. Guan R, Zheng W, Xu M. A retrospective analysis of the clinicopathologic characteristics of uterine cellular leiomyomas in China. Int J Gynaecol Obstet 2012;118:52-55.

8. Shiota M, Kotani Y, Umemoto M, Tobiume T, Hoshiai H. Recurrence of uterine myoma after laparoscopic myomectomy: what are the risk factors? Gynecol Minim Invasive Ther 2012;1:34-36.

9. Lonnerfors C, Persson J. Pregnancy following robot-assisted laparoscopic myomectomy in women with deep intramural myomas. Acta Obstet Gynecol Scand 2011;90:972-977.

10. Bedient CE, Magrina JF, Noble BN, Kho RM. Comparison of robotic and laparoscopic myomectomy. Am J Obstet Gynecol 2009;201:566.e1-566.e5. 